\title{
The State of Hydrogen in $\mathrm{Nb}$-based $\mathrm{Nb}-\mathrm{Mo}$ Alloys Analyzed by the Channelling Method
}

\author{
Eiichi YAGI \\ The Institute of Physical and Chemical Research (RIKEN), Wako, Saitama 351-0198 Japan, also at The School of Science and \\ Engineering, Waseda University, Okubo, Shinjuku-ku, Tokyo 169-8555 Japan.
}

(Received on August 31, 2002; accepted in final form on October 24, 2002)

\begin{abstract}
In order to locate hydrogen dissolved in metals a channelling method utilizing a nuclear reaction ${ }^{1} \mathrm{H}\left({ }^{11} \mathrm{~B}\right.$, $\alpha) \alpha \alpha$ with a ${ }^{11} \mathrm{~B}$ beam has been developed. This method has been applied to study the effect of metallic solutes on the state of hydrogen in metals. The lattice location of hydrogen in Nb-based Nb-Mo alloys with different concentrations $\left(C_{\mathrm{Mo}}\right)$ of undersized Mo atoms of 3, 10, 20 and 26 at \% has hitherto been investigated. These results are summarized in this paper and the interaction of hydrogen with metal solutes is discussed. At low Mo concentrations less than 10 at\% hydrogen is trapped by Mo atoms at room temperature due to attractive interaction to be located at sites displaced from tetrahedral ( $T$ ) sites by about $0.6 \AA$ toward the nearest neighbour Mo atom. In the case of $C_{\mathrm{Mo}}=3$ at $\%$, hydrogen is detrapped at $373 \mathrm{~K}$ and enter a $T$ site. The number of $\mathrm{H}$ atoms trapped by one $\mathrm{Mo}$ atom is limited. It is $0.7-1.0$ for $C_{\mathrm{Mo}}=3$ at $\%$ and decreases with increasing $C_{\mathrm{Mo}}$. The excess $\mathrm{H}$ atoms are located at $T$ sites. For $C_{\mathrm{Mo}}=20$ at $\%$ such trapped site occupancy is not observed, and $70-80 \%$ of the $\mathrm{H}$ atoms are located at $T$ sites and the rest are at octahedral (O) sites at low $\mathrm{H}$ concentrations. For $C_{\mathrm{Mo}}=26$ at\%, most of the $\mathrm{H}$ atoms are located at $T$ sites.
\end{abstract}

KEY WORDS: hydrogen; lattice location; channelling analysis; solute atoms; Nb-Mo alloy.

\section{Introduction}

In order to elucidate the mechanism of hydrogen embrittlement, knowledge of the fundamental properties of hydrogen such as its lattice location, its interaction with impurities, the behaviour in an atomic scale under stress, etc. is highly required. However, such knowledge is still insufficient, because the experimental methods for hydrogen detection are limited. Information on these properties is also important in the understanding of the hydrogen-related problems in the solid state physics. As to the lattice location of hydrogen isotopes, a number of experiments have been performed on deuterium by the neutron diffraction method and also by the channelling method with a ${ }^{3} \mathrm{He}$ beam, whereas on hydrogen there had been only a few neutron diffraction experiments because of their experimental difficulties and no channelling experiments despite their usefulness, because the method used for deuterium is ineffective for hydrogen. The behaviour of hydrogen is not necessarily the same as that of deuterium as recognized from, for example, the difference in the phase diagram between $\mathrm{V}-\mathrm{H}$ and $\mathrm{V}-\mathrm{D}$ systems and the difference in the diffusivity between $\mathrm{H}$ and D. From this point of view, isotope effect experiments on hydrogen are indispensable. Therefore, to study the lattice location of hydrogen a nuclear reaction ${ }^{1} \mathrm{H}\left({ }^{11} \mathrm{~B}, \alpha\right) \alpha \alpha$ with a ${ }^{11} \mathrm{~B}$ beam of about $2 \mathrm{MeV}$ was applied to the channelling method. This method was demonstrated to be very useful and it has become possible to locate hydrogen dissolved in metals. ${ }^{1)}$ This method has been used to various studies. Hydrogen dissolved in group $\mathrm{V}_{\mathrm{a}}$ metals in the periodic table $(\mathrm{V}, \mathrm{Nb}$ and $\mathrm{Ta}$ ) was observed to be locat- ed at tetrahedral $(T)$ sites. $^{2-4)}$ It was demonstrated that hydrogen in $\mathrm{V}$ is extremely sensitive to stress and changes its location reversibly from a $T$ site toward octahedral $(O)$ sites by $0.44 \AA$ under application and release of stress below the elastic limit. ${ }^{2,5}$

In the present paper an interaction of hydrogen with solute atoms in metallic alloys is focused, and the results of studies hitherto made on the change of the lattice location of hydrogen in $\mathrm{Nb}-\mathrm{Mo}$ alloys with Mo concentration, $C_{\mathrm{Mo}}$, are reported. This interaction is one of the important factors that influence behaviour and the state of hydrogen in metallic alloys. However, microscopic information on such interactions has been extremely limited. One of the examples is the effect of alloying on the terminal solubility of hydrogen (TSH) in group $\mathrm{V}_{\mathrm{a}}$ metals $(\mathrm{V}, \mathrm{Nb}$ and $\mathrm{Ta})$. It has been reported that for undersized metal solutes, e.g., $\mathrm{Nb}$ in $\mathrm{Ta}$, and $\mathrm{Mo}$ in $\mathrm{Nb}$, the TSH increases rapidly with solute concentration up to a certain value, whereas for the oversized solutes, e.g., $\mathrm{Ta}$ in $\mathrm{Nb}$, and $\mathrm{Nb}$ in $\mathrm{V}$, the $\mathrm{TSH}$ increases less rapidly with solute concentration. ${ }^{6-11)}$ In order to understand the mechanism of this effect, the lattice location of hydrogen in such alloy systems is to be investigated. In the present study, an Nb-Mo alloy system has been chosen. This system forms a solid solution over the entire Mo concentration range, and has a bcc crystal structure. The $\mathrm{TSH}$ in $\mathrm{Nb}$ at room temperature is a few per cent, whereas the TSH in Mo is very low. Therefore, the systematic studies on the $C_{\mathrm{Mo}}$ dependence of the hydrogen site occupancy are desired. Hence, lattice location of hydrogen was investigated by the channelling method for Nb-based $\mathrm{Nb}-\mathrm{Mo}$ alloys with various Mo concentrations up to 26 at $\%$ Mo. Even for deuteri- 


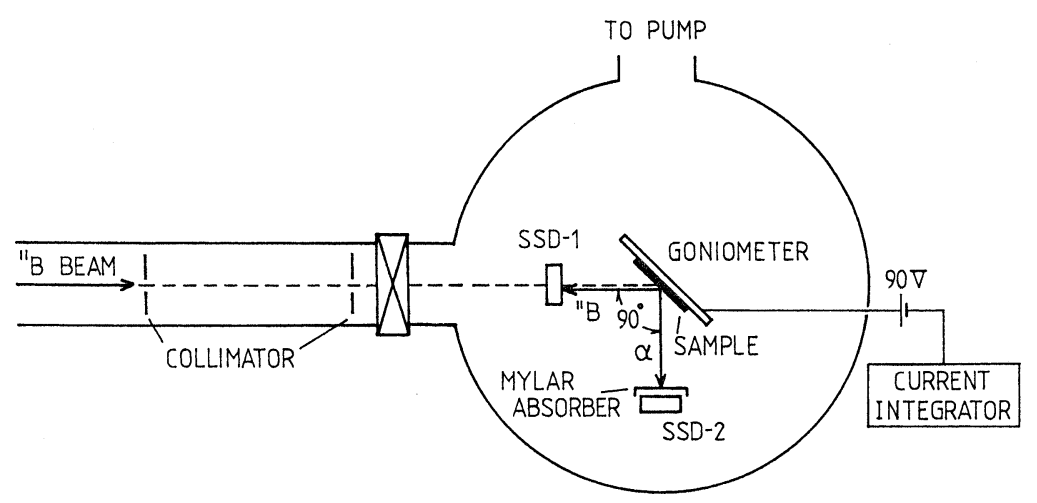

Fig. 1. Schematic diagram of the experimental set-up.

um, whose lattice location has been investigated extensively by both neutron and channelling methods, there has been only a few studies made in metallic alloys. ${ }^{12}$ The present study for Nb-Mo alloys with the same crystal structure as Fe will give helpful information to investigations of the state of hydrogen in iron and its alloys.

\section{Experimental Method}

The reason why the nuclear reaction ${ }^{1} \mathrm{H}\left({ }^{11} \mathrm{~B}, \alpha\right) \alpha \alpha$ was applied is as follows. In the detection of impurities by ion beams, usually the Rutherford backscattering spectroscopy (RBS) is used, but this method is not useful to the detection of hydrogen. Therefore, the nuclear reaction was utilized. This reaction exhibits a narrow resonance with a resonance width of about $66 \mathrm{keV}$ at about $1.8 \mathrm{MeV}$ of a ${ }^{11} \mathrm{~B}$ beam. ${ }^{13,14)}$ The energy of an incident ${ }^{11} \mathrm{~B}$ beam $(\sim 2 \mathrm{MeV})$ is reduced to about $1.8 \mathrm{MeV}$ at the depth of a few thousand $\AA$ in a specimen, and there the resonance reaction takes place effectively to emit $\alpha$ particles. Hydrogen can be detected by measuring these $\alpha$ particles, whose energy ranges from zero to about $5 \mathrm{MeV}$. To locate hydrogen, this hydrogen detection technique was combined with the channelling method.

Specimens are $\mathrm{Nb}$ and $\mathrm{Nb}-\mathrm{Mo}$ alloy single crystal slices whose largest face is perpendicular to the $\langle 110\rangle$ axis. They were annealed and then doped with hydrogen from the gas phase up to a desired concentration lower than the solubility limit. They were mounted on the specimen holder of the three-axis-goniometer. Channelling analyses were performed at room temperature for the $\langle 100\rangle$ and $\langle 110\rangle$ axial channels, the $\{100\}$ planar channel, and, if necessary, the $\{111\}$ planar channel at different spots on the same specimen with a ${ }^{11} \mathrm{~B}$ ion beam of about $2 \mathrm{MeV}$. The beam was collimated to the divergence of less than $0.076^{\circ}$. The beam spot was $1 \mathrm{~mm}$ in diameter and the intensity was $1.5-2.0$ $\mathrm{nA}$. The goniometer was tilted on the horizontal plane by about $45^{\circ}$ with respect to the incident beam. The yield of ${ }^{11} \mathrm{~B}$ ions backscattered by host metal atoms and the yield of the emitted $\alpha$ particles were measured as a function of the incident angle $\psi$ with respect to the channel direction in question by tilting the goniometer on the horizontal plane (channeling angular scan). Thus ${ }^{11} \mathrm{~B}$ - and $\alpha$-angular profiles were obtained. The backscattered ${ }^{11} \mathrm{~B}$ ions were detected at a scattering angle of $160^{\circ}$ in the vertical plane including the incident beam direction, while the $\alpha$ particles were detected at about $90^{\circ}$ with respect to the incident beam direction on the horizontal plane. In front of the detector for the $\alpha$ particles a $4-\mu$ m-thick Mylar foil was placed to eliminate the ${ }^{11} \mathrm{~B}$ ions which were scattered by the host metal atoms in the direction of $90^{\circ}$. The $\alpha$ particles can penetrate this foil to be detected. The experimental set-up is shown in Fig. 1.

If hydrogen is located at a site which is projected at the position $\mathbf{r}_{\mathrm{i}}$ in the channel on the plane perpendicular to the channel (a transverse plane) in question, the $\alpha$-angular profile exhibits peaks at angular positions $\psi_{\mathrm{p}}$, corresponding to the potential at the projected position, $\mathbf{r}_{i}$, due to flux-peaking effect in the spatial flux distribution of an incident beam in a channel under the channeling condition. ${ }^{15)}$ The position of a subpeak $\psi_{\mathrm{p}}$ is approximately given by

$$
\mathrm{E} \psi_{\mathrm{p}}{ }^{2}=U\left(\mathbf{r}_{\mathrm{i}}\right)
$$

$E$ is the incident energy of the beam and $U(\mathbf{r})$, which is the difference from its minimum value in the channel, is the continuum potential experienced by particles travelling in the channel. The origin for the position vector $\mathbf{r}$ is taken at the centre of the channel. Therefore, when hydrogen is located at a site that is projected at the centre of the channel, the $\alpha$-angular profile exhibits a central peak, while for hydrogen projected at off-centre positions, it exhibits off-centre peaks.

\section{Experimental Results}

\section{1. $\mathrm{Nb}$}

Figure 2 shows the channelling angular profiles obtained for the $\mathrm{Nb}$ specimen which was doped with hydrogen up to the hydrogen-to-metal-atom ratio $C_{\mathrm{H}}=0.023 .{ }^{16)}$ This specimen is in the $\alpha$ phase at room temperature. As the backscattering yields, those by atoms in the depth region from 60 to $180 \mathrm{~nm}$ were plotted. This region corresponds to the energy range where the resonance reaction in question takes place effectively. As the $\alpha$-particle yields, those with energies higher than $0.7 \mathrm{MeV}$ were plotted. (This is also the case for the $\mathrm{Nb}-\mathrm{Mo}$ alloy specimens.) The angular profiles of the $\alpha$-particle yield ( $\alpha$-angular profiles) exhibit a sharp central peak for the $\langle 100\rangle$ channel, a broad central peak consisting of three subpeaks at $\psi=0^{\circ}$ and around $\pm 0.35^{\circ}$ for the $\langle 110\rangle$ channel, and a small central peak superimposed on a shallow dip for the $\{100\}$ channel. In the inset the projections of $T$ and $O$ sites on the plane perpendicular to the channel in question are shown with open squares. The angular positions of subpeaks which are expected for the $T$-site occupancy are indicated by arrows. They are esti- 


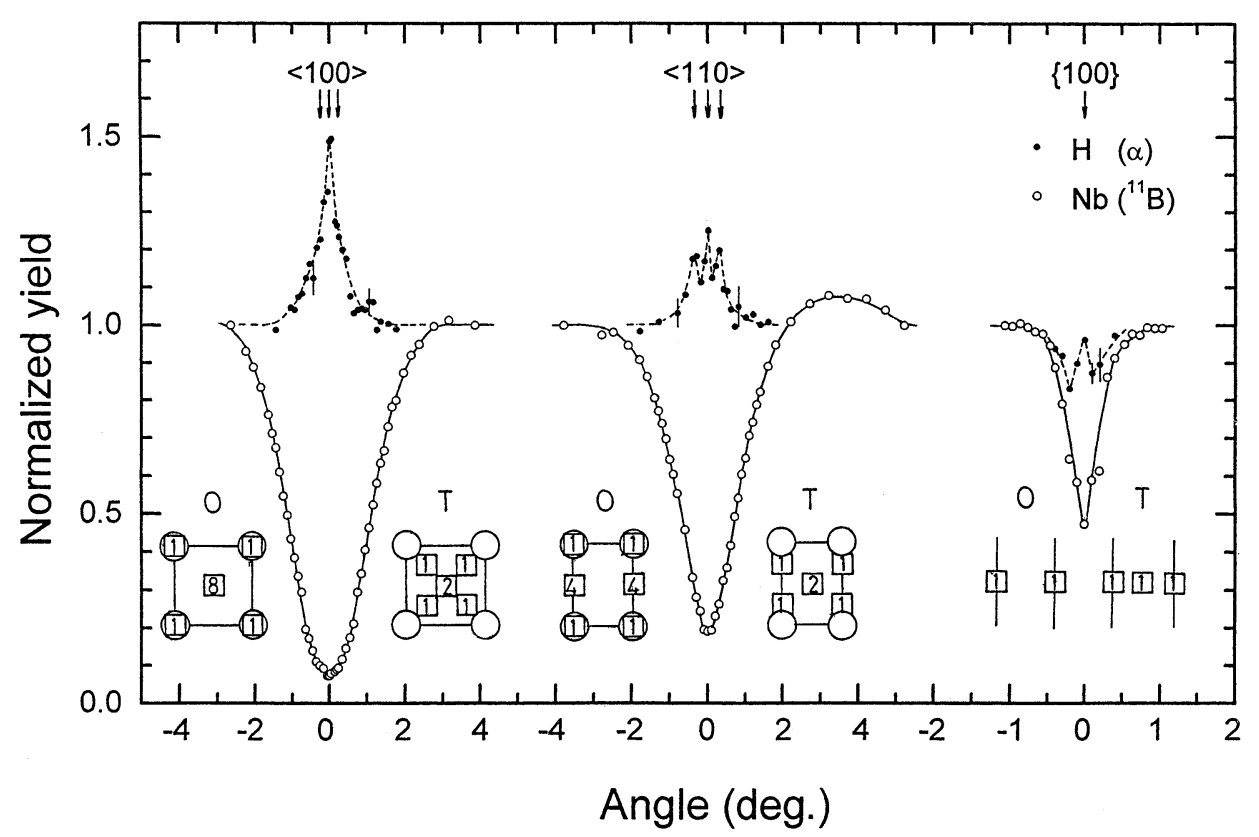

Fig. 2. Channelling angular profiles of $\alpha$-particle yield $(\bigcirc)$ and the yield of ${ }^{11} \mathrm{~B}$ ions backscattered by metal atoms $(\bigcirc)$, obtained at room temperature for $\mathrm{Nb}$ with hydrogen of $C_{\mathrm{H}}=0.023$. The full curves and the dashed curves have been drawn to guide the eye. In the insets the projections of $T$ and $O$ sites onto the plane perpendicular to the channel in question are shown by open squares with relative weights. The open circles and lines represent the projections of atomic rows and planes, respectively. The angular positions of subpeaks which are expected for the $T$ site occupancy are indicated by arrows.

mated using Eq. (1). Considering the beam divergence and the accuracy of angle setting with the goniometer, the angular resolution in the angular profile is about $\pm 0.05^{\circ}$. For the $\langle 100\rangle$ channel the expected three subpeaks are not well resolved, probably because of the expected small angular separation among them and the angular resolution, whereas for the $\langle 110\rangle$ and $\{100\}$ channels they are observed at the expected positions. These results suggest the $T$-site occupancy of hydrogen.

The lattice location of hydrogen was more definitely determined by comparing the observed $\alpha$-angular profiles with the calculated ones. As a first approximation, the calculation was made on the basis of the continuum potential model. For a beam with incident energy $E$ and an incident angle $\psi$, the beam flux $F$ at the impurity position $\mathbf{r}_{\mathrm{i}}$ in the channel on the transverse plane is given by ${ }^{17)}$

$$
\begin{aligned}
& F\left(\mathbf{r}_{\mathrm{i}}, E, \psi\right)=\iint_{A_{0}} f\left(\mathbf{r}_{\mathrm{i}}, E, \mathbf{r}_{\text {in }}\right) \mathrm{d} A\left(\mathbf{r}_{\text {in }}\right) \\
& f\left(\mathbf{r}_{\mathrm{i}}, E, \psi, \mathbf{r}_{\mathrm{in}}\right)=1 / A_{0} \quad U\left(\mathbf{r}_{\mathrm{TF}}\right) \leq E_{\perp}, \\
& =1 / A\left(E_{\perp}\right) \quad U\left(\mathbf{r}_{\mathrm{i}}\right) \leq E_{\perp}<U\left(\mathbf{r}_{\mathrm{TF}}\right), \\
& =0 \quad E_{\perp}<U\left(\mathbf{r}_{\mathrm{i}}\right) .
\end{aligned}
$$

In the calculation of a continuum potential $U(\mathbf{r})$, Erginsoy formula was used. ${ }^{18)} E_{\perp}$ is the transverse energy of an incident ion entering a channel at a position $\mathbf{r}_{\text {in }}$ with an incident angle $\psi$, and is given by $E_{\perp}=E \psi^{2}+U\left(\mathbf{r}_{\text {in }}\right) . A\left(E_{\perp}\right)$ is the accessible area for such ions having the transverse energy $E_{\perp}$, i.e., the area inside the calculated equipotential contour for $E_{\perp}$ in a transverse plane, and $A_{0}$ is the total area of a unit channel of the two dimensional real shape. $U\left(\mathbf{r}_{\mathrm{TF}}\right)$ is the potential at a distance of the Thomas-Fermi screening radius from atomic rows or planes.

For the above estimation of the flux distribution to be valid, it is required that statistical equilibrium in the spatial distribution of flux is established. The ions entering the crystal close to the axis or plane will not be able to pass through the centre of the channel before they penetrate a distance of the order of $\lambda=d_{\mathrm{p}} /\left(2 \psi_{1 / 2}\right)$, where $d_{\mathrm{p}}$ is the planar spacing of the channel and $\psi_{1 / 2}$ is the half angle of the channelling dip. In the case of the $\{100\}$ planar channel having the smallest $\psi_{1 / 2}$ among the three channels investigated, $\lambda$ is estimated to be about $19 \mathrm{~nm}$. Since there are a number of ions deflected by less than $\psi_{1 / 2}$, the equilibrium in the flux distribution will not be fully established until the ion beam penetrates distance much larger than $\lambda$. As the angular profiles shown in Figs. 2, 5 and 7 were obtained from backscattering yields and $\alpha$-particle yields associated with atoms in the region deeper than $60 \mathrm{~nm}$, this condition is considered to be fulfilled.

The normalized $\alpha$-particle yields $\chi_{\alpha}(\psi)$ were estimated from Eq. (2), considering that they are proportional to $F$. It was assumed that $\mathrm{H}$ atoms give the same angular profiles as those for the host metal atoms, i.e., the ${ }^{11} \mathrm{~B}$-angular profiles, when they are shadowed behind atomic rows or planes in the channel. In this calculation the beam divergence and a change in $E_{\perp}$ due to scattering by electrons were taken into account, but the effect of lattice vibration was not. The average increase in $E_{\perp}, \delta E_{\perp}$, was taken to be $1.8 \mathrm{eV}$. This corresponds to $\delta E_{\perp}$ during penetration of ions to the depth of $60 \mathrm{~nm}$ where the nuclear reaction cross section begins to increase. For comparison of the measured $\alpha$-angular profiles with the calculated ones, the theoretical $\alpha$-angular profiles for the perfect lattice were corrected in the following way by taking account of the random fraction of the incident beam arising from dechanneling during transmission through the surface region of the specimen. ${ }^{19)}$ The random fraction $f_{\mathrm{R}}(\psi)$ is given by 


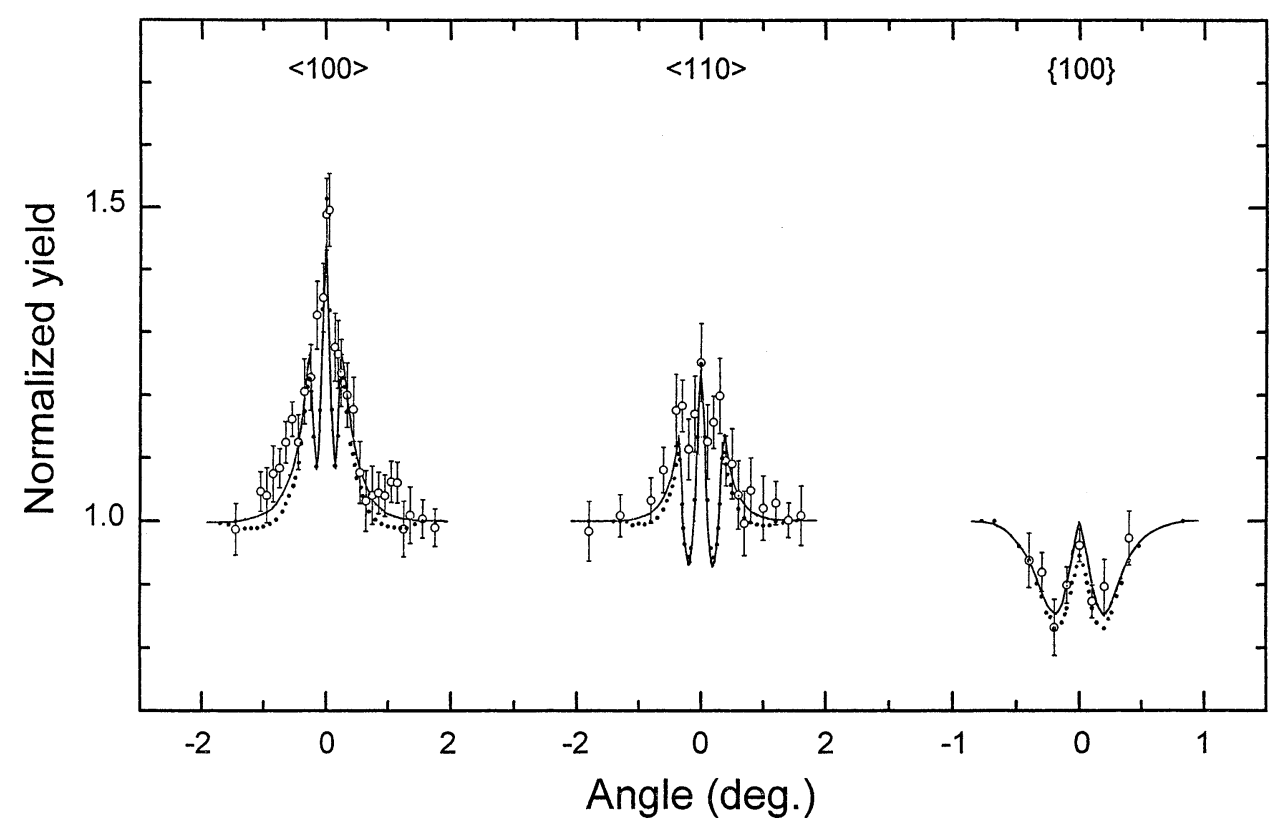

Fig. 3. The observed (open circles) and calculated $\alpha$-angular profiles for two kinds of hydrogen distributions for $\mathrm{Nb}$ with hydrogen of $C_{\mathrm{H}}=0.023 ; 100 \%$ of $\mathrm{H}$ atoms at $T$ sites (full curves) and $90 \%$ at T sites and $10 \%$ at $O$ sites (dotted curves).

$$
f_{\mathrm{R}}(\psi)=\left\{\chi_{\mathrm{B}}^{\exp }(\psi)-\chi_{\mathrm{B}}^{\text {theo }}(\psi)\right\} /\left\{1-\chi_{\mathrm{B}}^{\text {theo }}(\psi)\right\},
$$

and the channelled fraction is given by $1-f_{\mathrm{R}}(\psi) \cdot \chi_{\mathrm{B}}^{\exp }(\psi)$ and $\chi_{\mathrm{B}}^{\text {theo }}(\psi)$ represent the experimental values of normalized backscattering yields of $\mathrm{B}$ ions and the theoretical values calculated with Eq. (2) for the perfect lattice, respectively. The corrected yields $\chi_{\alpha}^{\text {corr }}(\psi)$ in the $\alpha$-angular profiles are obtained from the corresponding yields $\chi_{\alpha}^{\text {theo }}(\psi)$ according to

$$
\chi_{\alpha}^{\text {corr }}(\psi)=\chi_{\alpha}^{\text {theo }}(\psi)\left\{1-f_{\mathrm{R}}(\psi)\right\}+f_{\mathrm{R}}(\psi) .
$$

The comparison between the observed $\alpha$-angular profiles and those thus calculated with Eq. (4) for the $T$-site occupancy is shown in Fig. 3. In the calculated profiles the subpeaks are sharply resolved in contrast to the observed ones. In the above calculation hydrogen is treated as a point charge. Taking account of the extent of the wave function of hydrogen, thermal vibration, the angular resolution and the experimental statistical error, the subpeaks are not expected to be so sharply resolved. It is to be noted, however, that the observed peak positions are well reproduced by the calculated ones, which indicates the $T$-site occupancy of hydrogen. On the basis of the observed angular positions of subpeaks in three different channels, there are no possibilities that a major portion of $\mathrm{H}$ atoms are located at sites different from $T$ sites. For later discussions, the $\alpha$-angular profiles calculated for another distribution of the $\mathrm{H}$ atoms, $90 \%$ of them at $T$ sites and $10 \%$ at $O$ sites, are indicated by dotted curves. From comparison between the observed results and the calculated ones, this distribution cannot be completely excluded on the basis of the experimental accuracy. Thus, it is concluded that more than $90 \%$ of the $\mathrm{H}$ atoms are located at $T$ sites in $\mathrm{Nb}$.

\subsection{7 at $\% \mathrm{Nb}-3 \mathrm{at} \% \mathrm{Mo}$ and $90 \mathrm{at} \% \mathrm{Nb}-10 \mathrm{at} \% \mathrm{Mo}$ al- loys}

In the 3 at $\%$ Mo alloys, for $C_{\mathrm{H}}=0.02$, which is lower than
$C_{\mathrm{Mo}}$, the $\alpha$-angular profiles obtained for $\langle 100\rangle,\langle 110\rangle$ and $\{100\}$ channels at room temperature were completely different from those for hydrogen in $\mathrm{Nb}^{20)}$ The $\alpha$-angular profiles did not exhibit a peak at $\psi=0^{\circ}$ for all the three channels, but exhibited a small peak at $\psi=0.1^{\circ}$ for the $\langle 100\rangle$ channel, small off-centre peaks at $\psi \simeq \pm 0.2^{\circ}$ and $-0.6^{\circ}$ for the $\langle 110\rangle$ channel, and only a shallow dip for the $\{100\}$ channel. Hence, most of the $\mathrm{H}$ atoms are considered to be located at sites different from $T$ sites. When the hydrogen concentration was higher than $C_{\mathrm{Mo}}$, i.e., $C_{\mathrm{H}}=0.05$, the $\alpha$ angular profiles obtained at room temperature markedly changed from those for $C_{\mathrm{H}}=0.02$. The $\langle 100\rangle \alpha$-angular profile exhibited a higher central peak at $\psi=0^{\circ}$. In the $\langle 110\rangle$ channel, higher subpeaks newly appeared at $\psi=0^{\circ}$ and around $\pm 0.35^{\circ}$, and a small peak at $-0.6^{\circ}$ for $C_{\mathrm{H}}=0.02$ was still present. The $\{100\} \alpha$-dip became deeper, and a small central peak appeared to be superimposed on the dip. These results suggest that some portion of the $\mathrm{H}$ atoms are located at $T$ sites and the rest at the same sites as those observed for $C_{\mathrm{H}}=0.02$. After the measurement at room temperature, the specimen with $C_{\mathrm{H}}=0.05$ was measured at $373 \mathrm{~K}$. In the $\langle 100\rangle \alpha$-angular profile the central peak became higher, and for the $\{100\}$ channel a central peak superimposed on the dip became more conspicuous. These profiles are characteristic of the $T$-site occupancy. These results can be interpreted as follows. In the alloy with $C_{\mathrm{H}}=0.02$, most of the $\mathrm{H}$ atoms are trapped by Mo atoms at room temperature and located at sites different from $T$ sites (trapped sites $T_{\text {tr }}$ ). In the alloy with $C_{\mathrm{H}}=0.05$, some portion of hydrogen atoms are located at $T_{\mathrm{tr}}$ sites and the rest are at $T$ sites at room temperature, while at $373 \mathrm{~K}$ the trapped hydrogen atoms are detrapped and enter $T$ sites.

In order to determine the lattice location of the $T_{\mathrm{tr}}$ sites, the $\alpha$-angular profiles were calculated, considering five possible kinds of sites and that all $\mathrm{H}$ atoms are located at only one kind of these sites. Thus calculated results were compared with the observed ones. The observed $\alpha$-angular pro- 


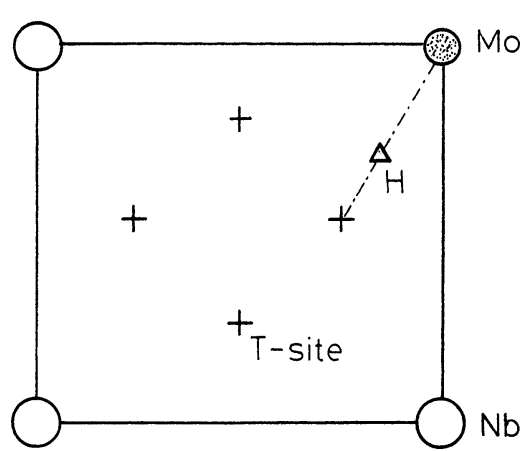

Fig. 4. A lattice location for a trapped hydrogen atom on a $\{100\}$ plane $(\triangle)$.

files obtained for $C_{\mathrm{H}}=0.02$ were well reproduced by the calculation made for the site displaced from a $T$ site toward the nearest neighbour lattice point by $0.62 \AA$, which is about $34 \%$ of their original separation (Fig. 4). ${ }^{20)}$ Hence, it is concluded that in the 3 at $\%$ Mo alloys with $C_{\mathrm{H}}=0.02$ all $\mathrm{H}$ atoms are trapped at room temperature by Mo atoms and are located at sites displaced from $T$ sites by about $0.6 \AA$ toward the Mo atoms at the nearest neighbour substitutional sites, and that at $373 \mathrm{~K}$ they are detrapped and enter $T$ sites. Admittedly, there is no way to determine directly the hydrogen position relative to Mo atoms, but, in view of the smaller size of an Mo atom in comparison to an $\mathrm{Nb}$ atom, the $T_{\text {tr }}$ site nearest to an Mo atom is believed to be most probable. The result that only a portion of the $\mathrm{H}$ atoms are trapped for $C_{\mathrm{H}}=0.05$ indicates that hydrogen trapping by Mo atoms has already been saturated. From the comparison of $\alpha$-angular profiles for $\mathrm{H}$ in $\mathrm{Nb}$ and the $3 \mathrm{at} \%$ Mo alloys with $C_{\mathrm{H}}=0.02$ and 0.05 , the number of hydrogen atoms trapped by one Mo atom, $\gamma$, can be roughly estimated to be $0.67-1.0 .^{20)}$ Hence, the binding energy between hydrogen and an Mo atom was roughly estimated to be $0.05 \mathrm{eV}^{20}$ ) in reasonable agreement with the previous determination by other technique. ${ }^{6}$

In the 10 at $\%$ Mo alloys the $\alpha$-angular profiles were similar to those for the $3 \mathrm{at} \%$ Mo alloys with $C_{\mathrm{H}}=0.05$. It was concluded that $\mathrm{H}$ atoms are also distributed over $T$ and $T_{\text {tr }}$ sites, but the magnitude of $\gamma$ is reduced to $0.25-0.35 .^{21,22)}$

\subsection{0 at $\%$ Nb-20 at $\%$ Mo Alloys}

In the 20 at $\%$ Mo alloy, channelling experiments were performed at room temperature for three different hydrogen concentrations $C_{\mathrm{H}}=0.026,0.042$ and $0.08 .{ }^{23)}$ The results are shown in Fig. 5. For $C_{\mathrm{H}}=0.026$ and 0.042 , the $\alpha$-angular profiles exhibit a large and sharp central peak superimposed on a shallow dip for the $\langle 100\rangle$ channel, a broad central peak consisting of three subpeaks at $\psi=0^{\circ}$ and around $\pm 0.35^{\circ}$ superimposed on a shallow dip for the $\langle 110\rangle$ channel, and a small central peak superimposed on a shallow dip for the $\{100\}$ channel. Except for the $\langle 100\rangle$ and $\langle 110\rangle$ shallow dip, they are similar to those observed for $\mathrm{H}$ in $\mathrm{Nb}$ (Fig. 2). These results suggest that a large portion of $\mathrm{H}$ atoms are located at $T$ sites and some portion of them are at sites which give the shallow dip in the $\langle 100\rangle$ and $\langle 110\rangle \alpha$-angular profiles, i.e., which are shadowed behind $\langle 100\rangle$ and $\langle 110\rangle$ atomic rows. These sites are considered to be $O$ sites, because one third of $O$ sites are shadowed behind $\langle 100\rangle$ and $\langle 110\rangle$ atomic rows (inset of Fig. 5), whereas $T$ sites are not. For $C_{\mathrm{H}}=0.08$, similar peaks are still observed for the three channels, but the shallow dips for the $\langle 100\rangle$ and $\langle 110\rangle$ channels became much shallower or almost non-existent, and, in addition, for the $\{100\}$ channel the relative depth of the $\alpha$ dip with respect to the ${ }^{11} \mathrm{~B}$-dip became smaller than those in the case of $C_{\mathrm{H}}=0.026$ and 0.042 . This result suggests that for $C_{\mathrm{H}}=0.08$ the fraction of the $\mathrm{H}$ atoms located at $O$ sites is significantly reduced and most of the $\mathrm{H}$ atoms are at $T$ sites.

The lattice location of hydrogen and its distribution in the 20 at $\%$ Mo alloy are determined by fitting the calculated $\alpha$-angular profiles to the observed ones. ${ }^{23)}$ Examples of $\alpha$ angular profiles calculated for various distributions of $\mathrm{H}$ atoms over $T$ and $O$ sites are shown in Fig. 6 together with experimental results. Taking account of the statistical error, for $C_{\mathrm{H}}=0.026$ and 0.042 the observed $\alpha$-angular profiles for the three channels are rather well reproduced by the $\mathrm{H}$ distribution of $70-80 \%$ at $T$ and $20-30 \%$ at $O$ sites. On the other hand, for $C_{\mathrm{H}}=0.08$, they are reproduced by the $\mathrm{H}$ distribution of $90-100 \%$ at $T$ sites and $0-10 \%$ at $O$ sites. It indicates that at high $\mathrm{H}$ concentrations the fraction of the $O$ site occupancy markedly decreases. ${ }^{23)}$ The $T_{\text {tr }}$-site occupancy was not observed, i.e., $\gamma=0$.

\subsection{4at\%Nb-26at\%Mo Alloys}

The channeling angular profiles for more concentrated 26 at $\%$ Mo alloys with different concentrations of hydrogen are shown in Fig. 7. ${ }^{16)}$ Irrespective of the hydrogen concentration the $\alpha$-angular profiles are very similar to those for $\mathrm{H}$ in $\mathrm{Nb}$ in Fig. 2. As distinct from the results on $\mathrm{H}$ in the 20 at $\%$ Mo alloys with low concentration of hydrogen, ${ }^{23)}$ a shallow dip is not observed for the $\langle 100\rangle$ and $\langle 110\rangle$ channels. The lattice location of hydrogen was determined by comparing the observed $\alpha$-angular profiles with the calculated ones. ${ }^{16)}$ It can be said that as in the case of hydrogen in $\mathrm{Nb}$ the observed profiles are well reproduced by the calculated ones for the $T$-site occupancy, although the subpeaks are not observed to be sharply resolved ones. The possible occupancy of $10 \% \mathrm{H}$ atoms at $O$ sites cannot be excluded, however, because the $\langle 100\rangle$ and $\langle 110\rangle \alpha$-angular profiles calculated for this distribution do not give a shallow dip so clearly as in the case of $20-30 \% \mathrm{H}$ atoms at $O$ sites. ${ }^{23)}$ It is, therefore, concluded that in the 26 at $\%$ Mo alloys $\mathrm{H}$ atoms are mostly located at $T$ sites and, the fraction of the $O$-site occupancy is at most $10 \%$. The $T_{\text {tr }}$-site occupancy was not observed. The site occupancy of hydrogen that has hitherto been observed in $\mathrm{Nb}-\mathrm{Mo}$ alloys is summarized in Table 1. The dependence of the number of $\mathrm{H}$ atoms trapped per one Mo atom, $\gamma$, on $C_{\mathrm{Mo}}$ is shown in Fig. 8.

\section{Discussion}

Previously it was reported that the halfwidth of X-ray reflection lines in $5 \mathrm{at} \%$ Mo and $5 \mathrm{at} \% \mathrm{~V}$ alloys ${ }^{6)}$ and the halfwidth of ${ }^{93} \mathrm{Nb}$ NMR absorption curves in 5 at $\% \mathrm{~V}$ and 8 at $\% \mathrm{~V}$ alloys ${ }^{24)}$ remarkably decreased with increasing $\mathrm{H}$ concentration, when the $\mathrm{H}$ concentration was less than the metal solute concentration. This result was interpreted as a result of the relaxation of internal strain induced by the undersized metal solutes, Mo and V, by hydrogen occupying their neighbouring interstitial sites, i.e., the trapping of hydrogen by solute atoms due to elastic interaction between 


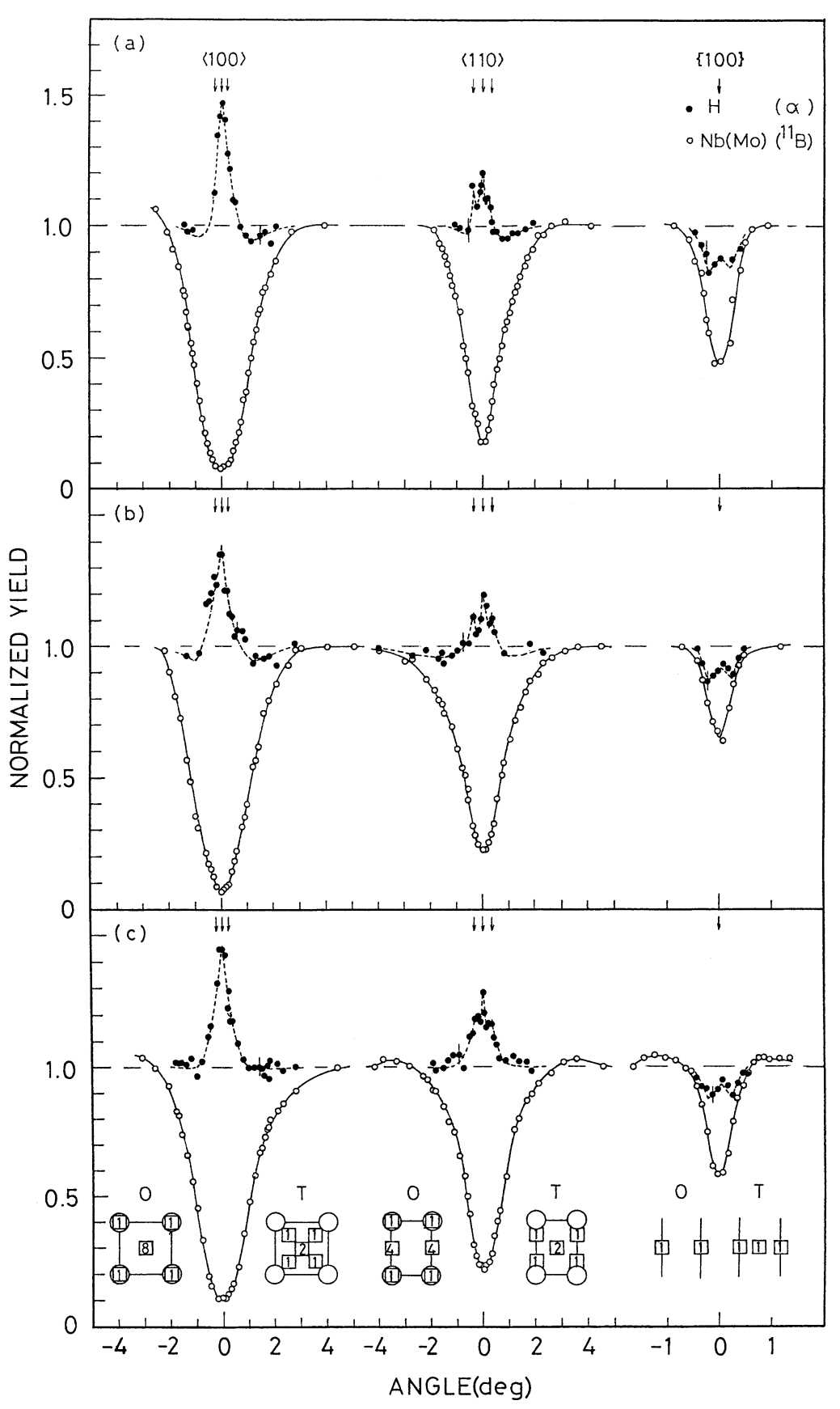

Fig. 5. Channelling angular profiles of $\alpha$-particle yield $(\bullet)$ and the yield of ${ }^{11} \mathrm{~B}$ ions backscattered by metal atoms $(\bigcirc)$, obtained at room temperature for the 20 at $\%$ Mo alloys with hydrogen of (a) $C_{\mathrm{H}}=0.026$, (b) 0.042 and (c) 0.08 . The full curves and the dashed curves have been drawn to guide the eye. The angular positions of subpeaks which are expected for the $T$-site occupancy are indicated by arrows.

them.

The site change of hydrogen in the $\mathrm{Nb}-\mathrm{Mo}$ alloys containing Mo less than 20 at $\%$ has been explained as follows. ${ }^{23)}$ At low $C_{\mathrm{Mo}}, \mathrm{H}$ atoms occupy well-defined trapped sites $\left(T_{\mathrm{tr}}\right.$-sites $)$ near Mo atoms due to the attractive elastic interaction. With increasing $C_{\mathrm{Mo}}$, the interference of the strain field induced by nearby Mo atoms changes the energy of the trapped sites, leading to a distribution of local atomic configurations and concomitant distribution of interstitial site energies. Correspondingly, trapping behaviour will become less well-defined, and, therefore, $\gamma$ becomes smaller. At higher $C_{\mathrm{Mo}}, 20 \mathrm{at} \% \mathrm{Mo}$, the average separation between Mo atoms becomes even smaller, and the effect of strain fields of individual Mo atoms would tend to average out. It is then considered more appropriate to regard this alloy as a pure metal having a homogeneously reduced lattice parameter.

A theoretical calculation on $\mathrm{H}$ in group $\mathrm{V}_{\mathrm{a}}$ metals by Sugimoto and Fukai ${ }^{25)}$ has shown that as the lattice is elongated along one of its cube axes, e.g., [001] axis, the wave function of hydrogen, originally localized on one of the four $T$ sites around the $O$ site on the (001) plane $\left(O_{\mathrm{z}}\right.$ site), is gradually displaced toward the $O_{\mathrm{z}}$ site $(\mathrm{d}-T$ site) and, after going through a resonant state of these four $\mathrm{d}-T$ configurations (4T), finally attains the $O_{z}$ site. (The symmetry of the $4 T$ configuration allows for the $O$-site occupancy as a particular case.) The energy difference between $T$ and $4 T$ configurations, $\Delta E_{4 T-T}$, depends on metal species and decreases 


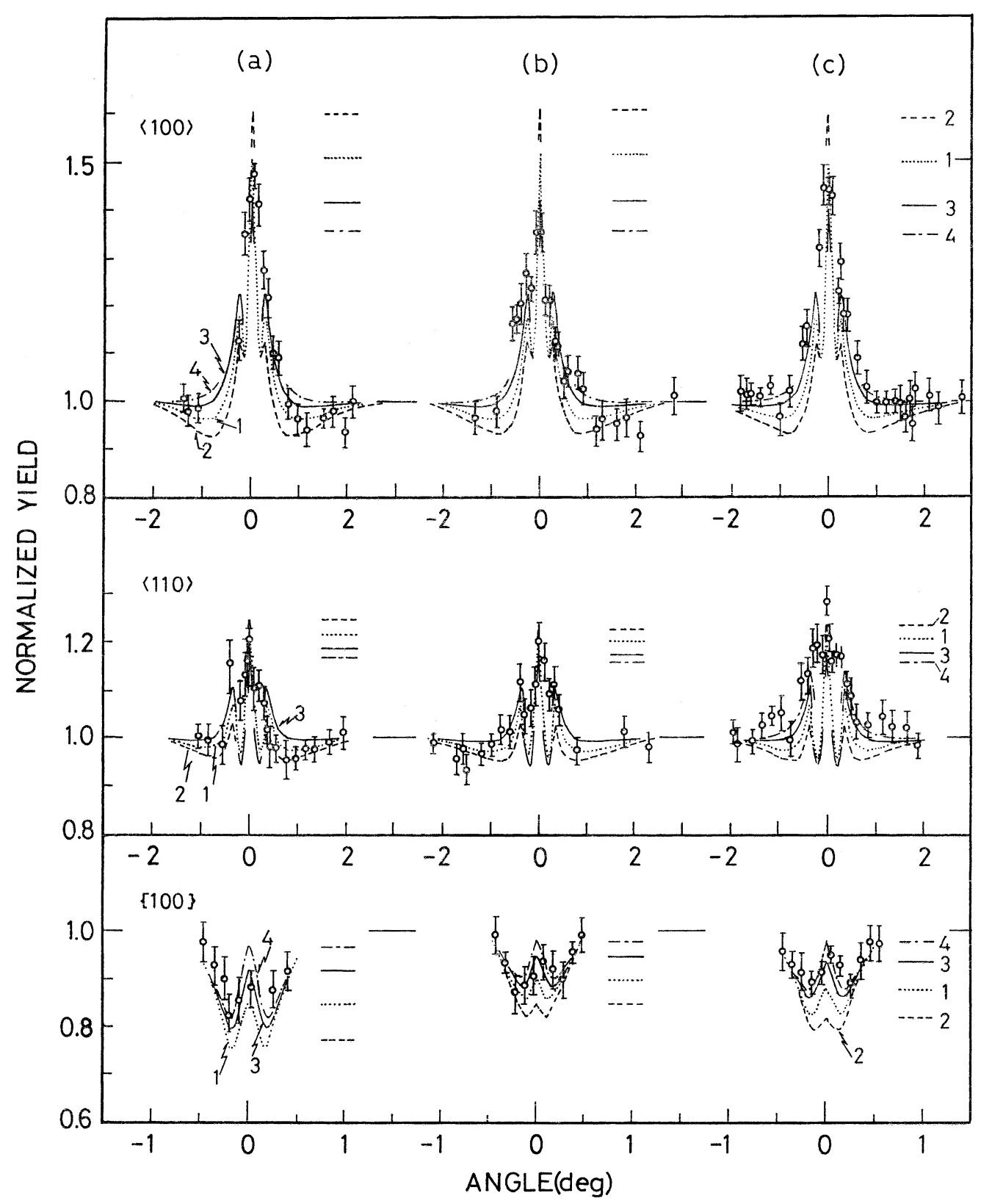

Fig. 6. The observed (open circles) $\alpha$-angular profiles and calculated ones for various hydrogen distributions over $T$ and $O$ sites for the 20 at $\%$ Mo alloys with hydrogen of (a) $C_{\mathrm{H}}=0.026$, (b) 0.042 and (c) 0.08 . Curve $1 ; 75 \%$ at $T, 25 \%$ at $O$, curve $2 ; 60 \%, 40 \%$, curve $3 ; 90 \%, 10 \%$, and curve $4 ; 100 \%, 0 \%$. Some of the curves are only partly shown, and the calculated yield for $\psi=0^{\circ}$ are indicated on the right hand side of the curves by horizontal lines of the type specific to each curve, because the figures are busy.

in order of $\mathrm{Ta}, \mathrm{Nb}$ and $\mathrm{V}$, i.e., with decreasing lattice parameter. The variation of $\Delta E_{4 T-T}$ with the relative size of an interstitial with respect to the host lattice arises from the fact that more drastic lowering of the potential energy of an $O$ site with outward displacement of its two nearest neighbour metal atoms occurs in more congested circumstances. Such a site change of $\mathrm{H}$ atoms from $T$ to $\mathrm{d}-T$ or $4 T$ configurations was observed by a channelling method in $\mathrm{V}$ under uniaxial stress, ${ }^{2,5)}$ although the stress level was much lower than that expected from the calculation. Such site changes were not observed in $\mathrm{Nb}$ and $\mathrm{Ta}^{3,4)}$

The appearance of the $O$-site occupancy in the $20 \mathrm{at} \%$ Mo alloys can be explained qualitatively by the size effect described above. On alloying with 20 at $\%$ Mo the lattice parameter of $\mathrm{Nb}$ is reduced by $0.8 \%$. Hence the energy differ- ence between $T$ and $O$ site becomes smaller, and $O$ sites become more easily available for occupation. The reduction of the fraction of the $O$-site occupancy, which was observed at a higher hydrogen concentration $\left(C_{\mathrm{H}}=0.08\right)$, is considered to be due to the interaction between $\mathrm{H}$ atoms, because the average separation between $\mathrm{H}$ atoms becomes smaller, $\sim 2.2 a$ ( $a$ : a lattice constant $){ }^{23)}$

However, the results on the 26 at $\%$ Mo alloys cannot be explained in a similar way by such size effects. Whereas the smaller lattice parameter of the 26 at $\%$ Mo alloys suggests the larger fraction of the $O$-site occupancy in the $26 \mathrm{at} \%$ Mo alloys, the experimental results indicate the contrary, which requires some additional considerations. With increasing $C_{\mathrm{Mo}}$, individual $T$ and $O$ sites have a variety of configurations of the nearest neighbour atoms, i.e., tetrahe- 


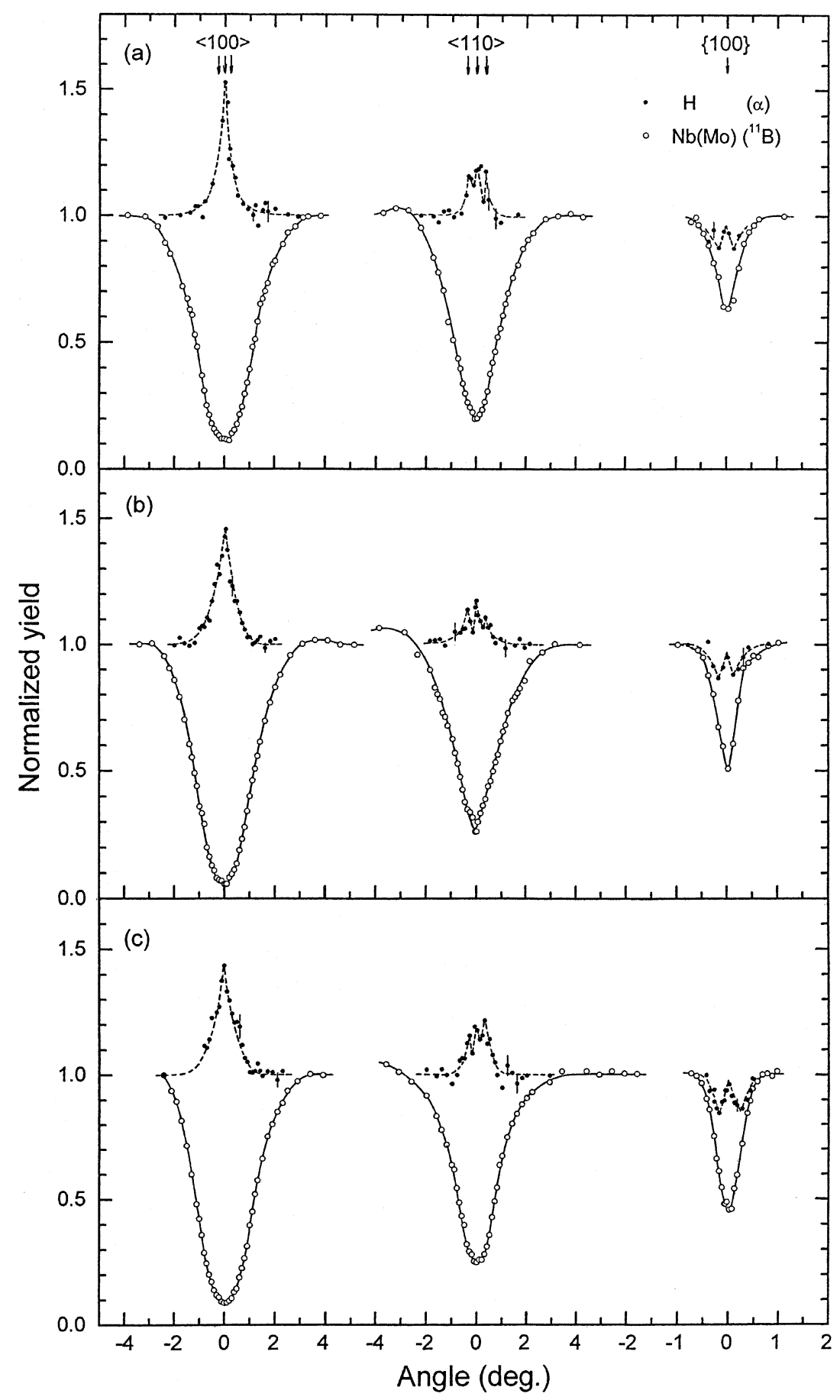

Fig. 7. Channelling angular profiles of $\alpha$-particle yield $(\bullet)$ and the yield of ${ }^{11} \mathrm{~B}$ ions backscattered by metal atoms $(O)$, obtained at room temperature for the 26 at $\%$ Mo alloys with hydrogen of (a) $C_{\mathrm{H}}=0.016$, (b) 0.026 and (c) 0.059 . The full curves and the dashed curves have been drawn to guide the eye. The angular positions of subpeaks which are expected for the $T$-site occupancy are indicated by arrows.

dra and octahedra comprising various numbers of Mo atoms. In the 26 at $\%$ Mo alloys there exists approximately one Mo atom per two unit cells on average. Therefore, all of the $T$ sites come to be surrounded by tetrahedra including one Mo atom as one of their constituent atoms on average, while each half of the $O$ sites come to be surrounded by octahedra including one Mo atom and two Mo atoms on average, respectively. Generally speaking, the interaction between metal solutes and hydrogen consists of electronic and elastic interactions. The electronic structure of $\mathrm{Nb}-\mathrm{Mo}$ alloys have been studied by angular correlation of positron annihilation radiation. The two dimensional angular corre- lation studies by West on $\mathrm{Nb}, 10$ at $\% \mathrm{Mo}, 30 \mathrm{at} \%$ Mo alloys and Mo clearly revealed the change of Fermi surface topology with increasing Mo concentration. ${ }^{26)}$ From the one dimensional angular correlation experiments on $20 \mathrm{at} \%$ Mo and 50 at $\%$ Mo alloys, Shiotani et al. demonstrated that extra valence electrons from Mo atoms fill the hole region in the momentum space and there exist well-defined Fermi surfaces even in the most concentrated alloy. ${ }^{27)}$ The result of the present experiments suggests that, in addition to a change in the electronic structure of alloys on alloying with higher concentration of Mo atoms, the change in the distribution of local atomic configurations as described above in- 


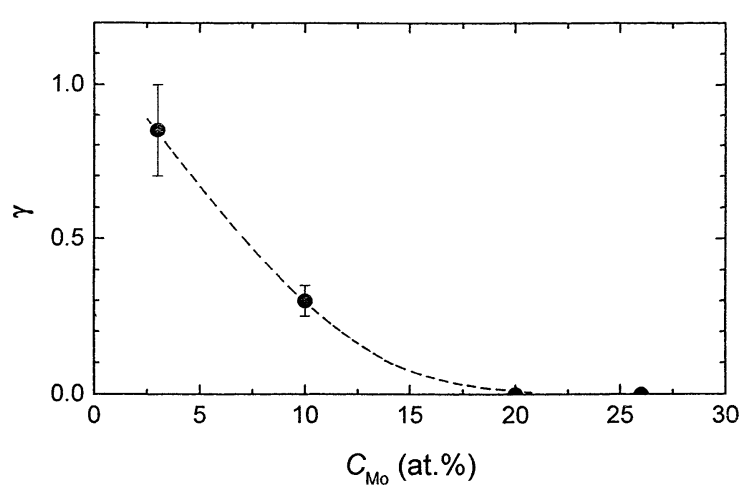

Fig. 8. The Mo concentration $\left(C_{\mathrm{Mo}}\right)$ dependence of the number of $\mathrm{H}$ atoms which are located at trapped $\left(T_{\text {tr }}\right)$ sites per one Mo atom, $\gamma$.

Table 1. The summary of the results on the site occupancy of hydrogen in $\mathrm{Nb}$-based $\mathrm{Nb}-\mathrm{Mo}$ alloys.

\begin{tabular}{|c|c|c|c|c|}
\hline \multirow{2}{*}{$\begin{array}{l}\text { Mo conc. } \\
C_{\mathrm{M}_{0}} \text { (at.\%) }\end{array}$} & \multirow{2}{*}{$\begin{array}{c}\mathrm{H} \text { conc. } \\
C_{\mathrm{H}}=[\mathrm{H}] /[\mathrm{M}]\end{array}$} & \multicolumn{3}{|c|}{ Sites of $\mathrm{H}$ atoms } \\
\hline & & (R.T.) & & $(373 \mathrm{~K})$ \\
\hline 0 & 0.023 & $T$ & $(*)$ & \\
\hline 3 & $\begin{array}{l}0.02 \\
0.05\end{array}$ & $\begin{array}{r}T_{\mathrm{tr}} \\
T_{\mathrm{tr}}(40-60 \%)+T\end{array}$ & $-60 \%)$ & $T$ \\
\hline 10 & 0.06 & $T_{\mathrm{tr}}(40-60 \%)+T$ & $-60 \%)$ & \\
\hline 20 & $\begin{array}{l}0.026 \\
0.042 \\
0.08\end{array}$ & $\begin{array}{l}T(70-80 \%)+C \\
T(70-80 \%)+O \\
T(90-100 \%)+O\end{array}$ & $\begin{array}{l}0-30 \%) \\
-30 \%) \\
10 \%)\end{array}$ & \\
\hline 26 & $\begin{array}{l}0.016 \\
0.026 \\
0.059\end{array}$ & $\begin{array}{l}T \\
T \\
T\end{array}$ & $\begin{array}{l}(*) \\
(*) \\
(*) \\
\end{array}$ & \\
\hline
\end{tabular}

*A possible $O$-site occupancy is less than $10 \%$. T; tetrahedral site,

$O$; octahedral site, $T_{\text {tr; }}$ trapped site (see text).

creases the energy difference between $T$ and $O$ sites and leads to the reduction in the fraction of the $O$-site occupancy. From the results on the lattice location of hydrogen it may be inferred, tentatively, that in the Nb-based $\mathrm{Nb}-\mathrm{Mo}$ alloys the elastic interaction is more important in the low $C_{\mathrm{Mo}}$ region, while in the high $C_{\mathrm{Mo}}$ region the contribution of electronic interaction becomes important. The experiment on the lattice location of hydrogen in more concentrated $\mathrm{Nb}-\mathrm{Mo}$ alloys is under consideration.

On the mechanism for the enhancement of the TSH on alloying with undersized metal solutes in group $\mathrm{V}_{\mathrm{a}}$ metals, a model on the trapping of hydrogen by solute atoms (the trapping model) has been proposed from X-ray diffraction and NMR experiments on $\mathrm{H}$ in $\mathrm{Nb}$-based $\mathrm{Nb}-\mathrm{Mo}$ and $\mathrm{Nb}-\mathrm{V}$ alloys. ${ }^{6,24)}$ On the other hand, Peterson and co-workers reported that evidence for trapping of $\mathrm{H}$ could not be observed in the studies on the isopiestic (equal hydrogen pressure) solubility of hydrogen and the hydrogen diffusion in $\mathrm{Nb}-\mathrm{V}$ alloys for a wide range of solute concentration and, therefore, the enhancement of the TSH could not be ascribed to a simple trapping of hydrogen. ${ }^{28,29)}$ Oates and Flanagan suggested that the enhancement of the TSH on alloying is likely to be fairly general and explained this by considering only the average effect of alloying on macroscopic thermodynamical quantities. ${ }^{30)}$ Therefore, the mechanism of the enhancement of the TSH is still an open question. From the studies on the lattice location of hydrogen, however, it is considered that in the $\mathrm{Nb}$-based $\mathrm{Nb}-\mathrm{Mo}$ alloy system the trapping of hydrogen by Mo solutes is effective for this enhancement of TSH, at least in the low concentration region of Mo solute atoms.

\section{Acknowledgements}

This research was financially supported in part by Special Coordination Funds of the Ministry of Education, Culture, Sports, Science and Technology, the Japanese Government. The author sincerely thanks Professor K. Nagamine, Professor M. Nagumo, Dr. T. Matsuzaki and Dr. Y. Yano for their support in various phases in performing this research. The author also thanks S. Nakamura, S. Koike, T. Matsumoto, T. Kobayashi, F. Kano, Y. Fukai, K. Watanabe, T. Osaka T. Urai, N. Tajima, K. Ogiwara and C. Kariya for their collaboration in various stages of this research.

\section{REFERENCES}

1) E. Yagi, T. Kobayashi, S. Nakamura, Y. Fukai and K. Watanabe: $J$. Phys. Soc. Jpn., 52 (1983), 3441.

2) E. Yagi, T. Kobayashi, S. Nakamura, Y. Fukai and K. Watanabe: Phys. Rev. B, 31 (1985), 1640.

3) E. Yagi, S. Nakamura, T. Kobayashi, K. Watanabe and Y. Fukai: J. Phys. Soc. Jpn., 54 (1985), 1855.

4) E. Yagi, S. Nakamura, T. Kobayashi, F. Kano, K. Watanabe, Y. Fukai and T. Osaka: J. Phys. Soc. Jpn., 55 (1986), 2671.

5) E. Yagi, T. Kobayashi, S. Nakamura, F. Kano, K. Watanabe, Y. Fukai and S. Koike: Phys. Rev. B, 33 (1986), 5121.

6) T. Matsumoto, Y. Sasaki and M. Hihara: J. Phys. Chem. Solids, 36 (1975), 215.

7) Y. Sasaki and M. Amano: Proc. of the Second Int. Cong. on Hydrogen in Metals, ed. by International Association for Hydrogen Energy, Pergamon Press, Oxford, (1977), $3 C_{3}$.

8) G. Cannelli and R. Cantelli: Proc. of the Sixth Int. Conf. on Internal Friction and Ultrasonic Attenuation in Solids, ed. by R. R. Hasiguti and N. Mikoshiba, Univ. Tokyo Press, Tokyo, (1977), 491.

9) D. G. Westlake and J. F. Miller: J. Less-Common Met., 65 (1979), 139.

10) J. F. Miller and D. G. Westlake: Proc. of the Second Japan Institute of Metals Int. Symp. on Hydrogen in Metals, (1979), Trans. Jpn. Inst. Met., 21, (1980), Suppl., 153.

11) S. Tanaka and H. Kimura: Trans. Jpn. Inst. Met., 20 (1979), 647.

12) H. Naramoto, K. Kawatsura, M. Sasaki, Y. Sugizaki, K. Ozawa, S. Yamaguchi, Y. Fujino and M. Aoki: Nucl. Instrum. Methods Phys. Res. B, 33 (1988), 595.

13) Von D. Denhard, D. Kamke and P. Kramer: Ann. Phys. (Germany), 14 (1964), 201.

14) E. Ligeon and A. Guivarch: Radiat. Eff., 22 (1974), 101.

15) J. U. Andersen, O. Andreasen, J. A. Davies and E. Uggerhøj: Radiat. Eff., 7 (1971), 25.

16) E. Yagi, S. Koike, T. Matsumoto, T. Urai, N. Tajima and K. Ogiwara: Phys. Rev. B, 66 (2002), 024206.

17) L. C. Feldman, J. Mayer and S. T. Picraux: Materials Analysis by Ion Channeling, Academic Press, New York, (1982), 73.

18) C. Erginsoy: Phys. Rev. Lett., 15 (1965), 360.

19) R. B. Alexander, P. T. Callaghan and J. M. Poate: Phys. Rev. B, 9 (1974), 3022.

20) E. Yagi, S. Nakamura, F. Kano, T. Kobayashi, K. Watanabe, Y. Fukai and T. Matsumoto: Phys. Rev. B, 39 (1989), 57.

21) E. Yagi, T. Kobayashi, K. Watanabe, Y. Fukai and T. Matsumoto: Proc. Jpn. Acad., 65B (1989), 38.

22) E. Yagi: Radiat. Eff. Defects Solids, 124 (1992), 21.

23) E. Yagi and S. Koike: J. Phys. Soc. Jpn., 67 (1998), 340.

24) T. Matsumoto: J. Phys. Soc. Jpn., 42 (1977), 1583.

25) H. Sugimoto and Y. Fukai: Phys. Rev. B, 22 (1980), 670.

26) R. N. West: Inst. Phys. Conf. Ser. No. 55, Physics of Transition Metals, Inst. Phys., London, (1980), 35.

27) N. Shiotani, T. Okada, H. Sekizawa and T. Nakamichi: J. Phys. Soc. Jpn., 52 (1983), 2858.

$28)$ D. T. Peterson and S. O. Nelson: Metall. Trans. A, 16 (1985), 367.

$29)$ D. T. Peterson and H. M. Herro: Metall. Trans. A, 17 (1986), 645.

30) W. A. Oates and T. B. Flanagan: Acta Metall., 33 (1985), 693. 